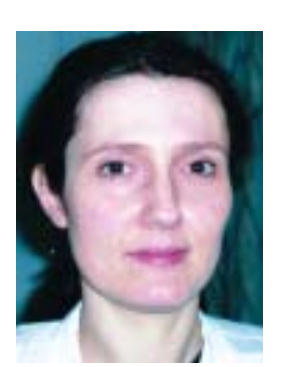

Katharina Eger

\title{
Erkrankungen mit Störung der neuromuskulären Übertragung
}

\author{
Katharina Eger \\ Klinik und Poliklinik für Neurologie der Martin-Luther-Universität Halle-Wittenberg, \\ Halle/Saale
}

psychoneuro 2003; 29 (3): 113-117

\section{Myasthenia gravis \\ Klinik}

Erstes Symptom der Myasthenia gravis kann ein nur uncharakteristisches Schwächegefühl sein, das bei Belastung zunimmt und nicht selten fälschlicherweise den Verdacht auf eine psychosomatische Erkrankung oder Depression lenkt. Aber auch ein rascher Beginn der Symptomatik mit deutlicher Schwäche ist möglich.

Unterschieden werden eine generalisierte Form und eine okuläre Form der Myasthenia gravis. Bei der rein okulären Form sind ausschließlich die äußeren Augenmuskeln betroffen. In ca. 50-60\% der Fälle beginnt die Myasthenie mit okulären Symptomen; bei bis zu $80 \%$ dieser Patienten kommt es innerhalb der folgenden drei Jahre zur Generalisierung der Symptomatik. Nur bei 10-20\% der Patienten wird eine rein okuläre Form beobachtet (2). Kennzeichnend sind eine Spontanptosis (meist asymmetrisch und beide $\mathrm{Au}$ gen wechselnd ausgeprägt betreffend) und Doppelbilder; am Morgen sind die Beschwerden typischerweise deutlich geringer oder gar nicht vorhanden und nehmen im Tagesverlauf - unter Belastung - zu.

Die bekanntesten Erkrankungen mit Störung der neuromuskulären Übertragung sind die Myasthenia gravis mit einem postsynaptischen und das Lambert-Eaton myasthene Syndrom (LEMS) mit einem präsynaptischen Defekt. Autoantikörper spielen bei beiden Erkrankungen eine wesentliche Rolle in der Pathogenese. Demgegenüber sind kongenitale myasthene Syndrome eine heterogene Gruppe erblicher Erkrankungen, die nicht autoimmun bedingt sind und deren Ursachen sowohl auf prä- als auch auf postsynaptischer Ebene liegen können. Die Myasthenia gravis zeigt sich mit typischen belastungsabhängigen Paresen, bevorzugt der proximalen Muskulatur, der bulbären Muskulatur oder der äußeren Augenmuskeln. Bei den kongenitalen myasthenen Syndromen sind die Paresen weniger deutlich belastungsabhängig. Das LEMS ist neben proximal betonten Paresen auch durch autonome Symptome wie Mundtrockenheit und Obstipation gekennzeichnet. Symptomatische Therapien wie Azetylcholinesterasehemmer bei der Myasthenia gravis oder 3,4-Diaminopyridin beim LEMS sowie immunsuppressive Therapien stellen heute die Mittel der Wahl dar bei der Myasthenia gravis und dem LEMS.

Die generalisierte Form der Myasthenia gravis kann ebenfalls asymmetrisch auftreten und schwerpunktmäßig bestimmte Muskelgruppen betreffen, z.B. die proximale Muskulatur der Extremitäten oder die pharyngeale Muskulatur. Der Patient beklagt dann z.B. bei zunehmender Belastung Probleme beim Treppensteigen, Haarewaschen, oder auch beim Schlucken, Kauen, Sprechen. Bedrohlich kann die Symptomatik werden, wenn durch die Schluckstörung Aspirationsgefahr oder bei Beteiligung der Atemmuskulatur die Gefahr einer Ateminsuffizienz besteht (myasthene Krise).

\section{Pathogenese}

Hervorgerufen wird die Myasthenia gravis durch Autoantikörper, die sich gegen den postsynaptischen Azetylcholinrezeptor (Abb. 1a, 1b) richten. Als Folge dessen werden eine Komplementaktivierung und konsekutiv die Verminderung oder Zerstörung der postsynaptischen Muskelzellmembran, ein gesteigerter Abbau von Azetylcholinrezeptoren, eine direkte Beeinflussung des Ionenkanals und auch eine direkte Blockade des Rezeptors durch den Antikörper vermutet (10).

Bei 3-4\% der Patienten findet sich eine familiäre Häufung der 
Myasthenie oder die Assoziation mit anderen Autoimmunerkrankungen (z.B. Autoimmunthyreopathie, juveniler Diabetes mellitus, Mb. Addison etc.). Die Assoziation zu bestimmten HLA-Merkmalen wurde beschrieben (z.B. HLA-B8, -DR3, -DQ- $\beta$ ).

Ein Zusammenhang zwischen Myasthenia gravis und Thymusveränderungen ist schon seit Ende des 19. Jahrhunderts bekannt. Bei ca. 10\% der Patienten lässt sich ein Thymom nachweisen, das semimaligne oder maligne sein kann. Andererseits entwickeln 30-61\% der Patienten mit einem Thymom eine Myasthenie. Bei den meisten Patienten, insbesondere bei Frauen unter dem 40. Lebensjahr, findet sich eine Thymushyperplasie („Thymitis“).

Wahrscheinlich spielt beim Thymom ein mit dem Azetylcholinrezeptor kreuzreagierendes Protein als Autoantigen eine Rolle. Bei der Thymushyperplasie hingegen werden histologisch Keimzentren als
Ausdruck eines aktiven immunologischen Prozesses gesehen; möglicherweise kommt den so genannten Myoidzellen eine bedeutende Rolle in der Pathogenese zu, die Azetylcholinrezeptoren exprimieren. Außerdem können B-Lymphozyten und Plasmazellen Azetylcholinrezeptoren exprimieren $(10,20)$.

\section{Diagnostik}

Die Azetylcholinrezeptor-Antikörper sind bei etwa 80 bis $90 \%$ der Patienten mit generalisierter Myasthenia gravis nachweisbar $(17,18)$; bei der okulären Form lässt sich der Antikörper nur in ca. 50\% nachweisen. Ein fehlender Azetylcholinrezeptor-Antikörper-Nachweis schließt also eine Myasthenia gravis keinesfalls aus. Die Höhe des AntikörperTiters korreliert nicht mit der Schwere der myasthenen Symptomatik. Ein Anstieg oder ein Rückgang des Titers geht beim selben Patienten jedoch oft parallel mit einer

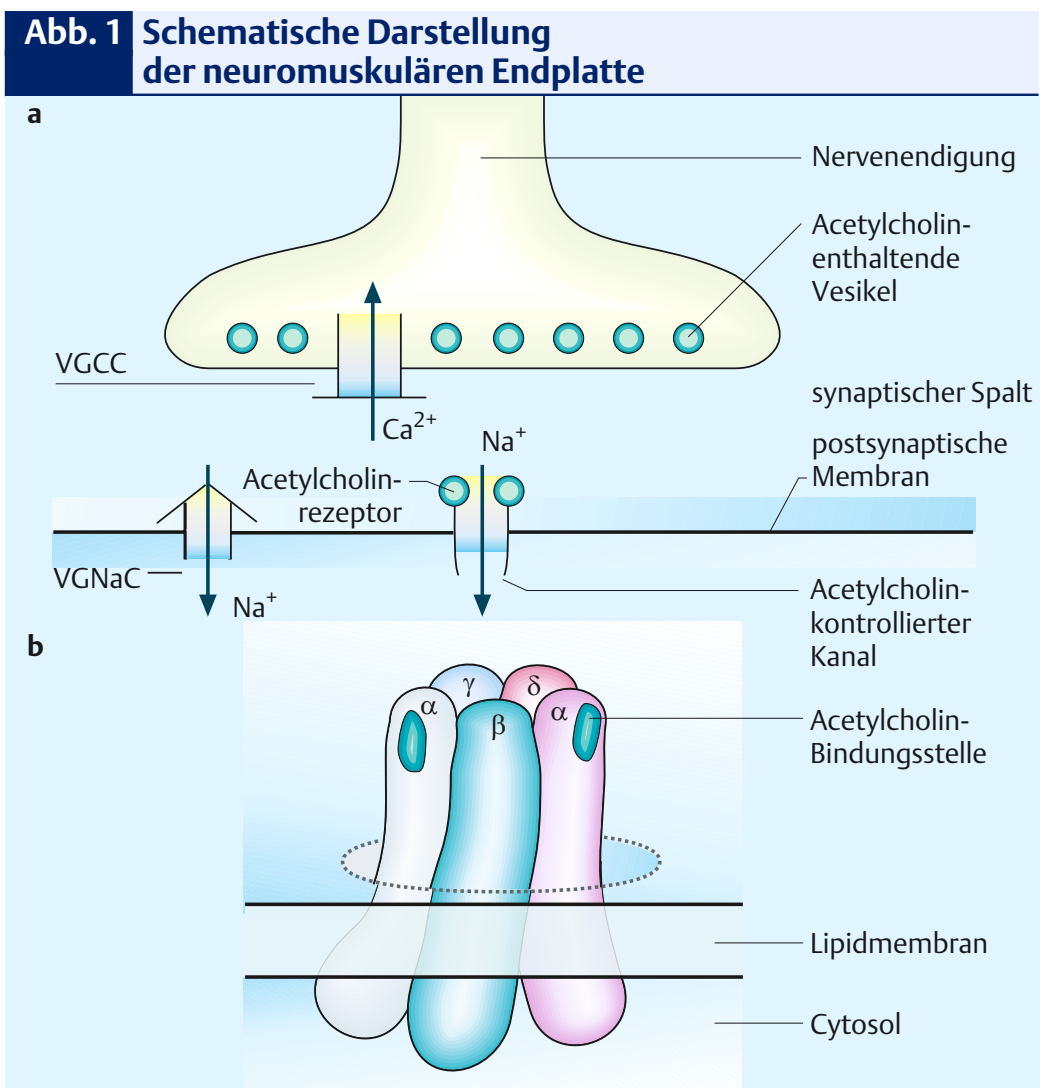

(VGCC = voltage gated calzium channel, spannungsabhängiger Kalzium-Kanal; $\mathrm{VGNaC}=$ voltage gated natrium channel, spannungsabhängiger Natrium-Kanal) a Übersicht; b Detailansicht des Azetylcholinrezeptors mit den $\alpha-, \beta-, \gamma$, und $\delta$ Untereinheiten.

Verschlechterung oder Verbesserung der Symptomatik einher.

Kürzlich konnte gezeigt werden, dass bei ca. zwei Drittel der „seronegativen“ (ohne Azetylcholinrezeptor-Nachweis) Patienten ein Antikörper vorkommt, der sich gegen die muskelspezifische Kinase (MuSK) richtet (6).

Antikörper gegen quergestreifte Muskulatur werden bei $80 \%$ der Patienten mit Thymom gefunden, sind jedoch nicht spezifisch für ein Thymom. Antikörper gegen Titin, ein Muskelprotein, sind bei bis zu über 90\% der Myasthenie-Patienten mit Thymom nachweisbar, aber bis zu 47\% auch bei Patienten mit Thymusinvolution, bis zu 6\% bei Thymushyperplasie $(1,4,19)$.

Elektrophysiologische Diagnostik ist insbesondere mit der repetitiven Stimulation von $3 \mathrm{~Hz}$ („MyasthenieTest“) und dem Nachweis eines pathologischen Dekrements wesentlicher Bestandteil der Diagnostik (siehe Beitrag von M. Kornhuber: „Diagnostische Möglichkeiten bei neuromuskulären Krankheiten“" in diesem Heft).

Der Tensilon-Test ${ }^{\circledR}$ ist für die Diagnosestellung vor allem dann entscheidend, wenn trotz typischer Anamnese und klinischem Befund die Azetylcholinrezeptor-Antikörper im Serum negativ sind. Edrophoniumchlorid (Tensilon ${ }^{\circledR}$ ) hemmt die Cholinesterase und führt zu einem größeren Angebot von Azetylcholin im synaptischen Spalt. Bereits wenige Sekunden nach intravenöser Applikation der Substanz wird eine deutliche Besserung der Symptomatik (z.B. der Ptosis) sichtbar.

Bildgebende Diagnostik umfasst die Computertomographie oder Magnetresonanztomographie mit der Frage, ob ein Thymom nachweisbar ist. Die Octreotid-Szintigraphie kann ergänzend bei der Suche nach einem Thymom eingesetzt werden (8).

\section{Therapie}

Unterschieden werden die rein symptomatische Therapie mit Cholinesterasehemmern und das Immunsystem beeinflussende Therapien wie Kortikosteroide, Azathioprin und Plasmapherese. Neue Immun- 
suppressiva wie Mycophenolat mofetil sowie die intravenöse Gabe von Immunglobulinen stellen eine Erweiterung der bisherigen therapeutischen Möglichkeiten dar.

Die Thymektomie wird bei Patienten mit generalisierter Form der Myasthenia gravis im Alter zwischen 10 und 65 Jahren und bei Patienten mit Thymom empfohlen (11). Remissionen wurden bei $60 \%$ der Patienten mit juvenil auftretender Myasthenie beschrieben (9). Bei isolierter okulärer Form wird die Indikation kontrovers gehandhabt. Gute Ergebnisse wurden hier vor allem bei jüngeren Patienten mit einer Krankheitsdauer unter zwei Jahren erzielt $(5,15,16)$. Schumm et al. (15) zeigten, dass keiner von 18 Patienten mit okulärer Myasthenie nach Thymektomie generalisierte Symptome entwickelte. Im Kontrast dazu hatten Kupersmith et al. (7) berichtet, dass nur drei von 32 Patienten (9\%) mit rein okulärer Symptomatik nach
Therapie mit Prednisolon generalisierte Symptome beklagten. Letztlich wird man die Indikation zur Thymektomie bei okulärer Form der Myasthenie auch vom Alter des Patienten und Begleiterkrankungen sowie vom Erfolg der medikamentösen Therapie abhängig machen.

\section{Kongenitale \\ Myasthenie-Syndrome}

Die kongenitalen MyasthenieSyndrome sind sehr selten. Den klinisch verschiedenen Formen liegen unterschiedliche Ursachen zugrunde, die entweder präsynaptisch z.B. Größe und Freisetzung der Azetylcholin-Vesikel betreffen oder postsynaptisch z.B. eine Veränderung der Funktion des Azetylcholinrezeptors bedingen; Beispiele sind in Tabelle 1 aufgeführt (Tab. 1).

Leitsymptome sind Ptose, Ophthalmoplegie und Schwäche der Gesichtsmuskulatur; proximal betonte Paresen werden häufig beobachtet.
Die für die Myasthenia gravis typische myasthene Symptomatik mit Kraftminderung im Verlauf körperlicher Belastung wird hier kaum beklagt. Ein hereditäres kongenitales myasthenes Syndrom kann sich sowohl in der frühen Kindheit als auch im höheren Erwachsenenalter manifestieren.

Azetylcholinrezeptor-Antikörper sind bei diesen Patienten nicht nachweisbar; durch den AntikörperNachweis gelingt in der Regel die Abgrenzung zur Myasthenia gravis.

Elektrophysiologische Untersuchungen wie die Einzel- und Serienstimulation sind in der Diagnostik der kongenitalen myasthenen Syndrome oft wegweisend (siehe Beitrag von M. Kornhuber in diesem Heft).

In speziellen Zentren können invitro-Untersuchungen, spezielle histochemische Methoden und letztlich die Suche nach der zugrunde liegenden Mutation durchgeführt werden, um die Diagnose zu sichern.

\section{Tab. 1 Kongenitale Myasthenie-Syndrome (Beispiele; nach 3)}

\begin{tabular}{|c|c|c|c|}
\hline & Krankheitsbezeichnung & Pathogenese & Erbmodus/Genort \\
\hline $\begin{array}{l}\text { präsynaptischer } \\
\text { Defekt }\end{array}$ & familiäre infantile Myasthenie & $\begin{array}{l}\text { Verringerung der mittleren Größe } \\
\text { der präsynaptischen, Azetylcholin } \\
\text { speichernden Vesikel }\end{array}$ & nicht bekannt \\
\hline \multirow[t]{4}{*}{$\begin{array}{l}\text { postsynaptischer } \\
\text { Defekt }\end{array}$} & $\begin{array}{l}\text { kongenitaler Azetyl- } \\
\text { cholinesterasemangel }\end{array}$ & $\begin{array}{l}\text { Mangel an Azetylcholinesterase im } \\
\text { synaptischen Spalt }\end{array}$ & $\begin{array}{l}\text { AR } \\
3 p 25 \\
\text { (mind. } 24 \text { versch. } \\
\text { Mutationen bekannt) }\end{array}$ \\
\hline & Slow-Channel-Syndrom & $\begin{array}{l}\text { Verlängerung der Öffnungsdauer } \\
\text { des lonenkanals des Azetylcholin- } \\
\text { Rezeptors }\end{array}$ & $\begin{array}{l}\text { AD } \\
\text { 2q24-32 ( } \alpha \text {-Untereinheit) } \\
\text { 17p13.1 ( } \beta \text {-Untereinheit) } \\
\text { 2q33-34 ( } \delta \text {-Untereinheit) } \\
\text { 17p13-p12 } \\
\text { ( } \epsilon \text {-Untereinheit) } \\
\text { (verschiedene Mutationen je } \alpha-\text {, } \\
\beta-, \delta-, \epsilon \text {-Untereinheit bekannt) }\end{array}$ \\
\hline & Fast-Channel-Syndrom & $\begin{array}{l}\text { Verkürzung der Öffnungsdauer des } \\
\text { lonenkanals des Azetylcholin- } \\
\text { rezeptors durch Mutationen in } \\
\text { Azetylcholinrezeptor-Untereinheiten }\end{array}$ & $\begin{array}{l}\text { AR } \\
\text { 2q24-32 ( } \alpha \text {-Untereinheit) } \\
\text { 2q33-34 ( } \delta \text {-Untereinheit) } \\
\text { 17p13-p12 ( } \epsilon \text {-Untereinheit) } \\
\text { (verschiedene Mutationen je } \alpha \text {-, } \\
\beta-, \delta-, \epsilon-U n t e r e i n h e i t \text { bekannt) }\end{array}$ \\
\hline & Azetylcholinrezeptor-Mangel & $\begin{array}{l}\text { Mangel an bestimmten } \\
\text { Untereinheiten des Azetyl- } \\
\text { cholinrezeptors }\end{array}$ & $\begin{array}{l}\text { AR } \\
\text { 2q24-32 ( } \alpha \text {-Untereinheit) } \\
\text { 17p13.1 ( } \beta \text {-Untereinheit) } \\
\text { 2q33-34 ( } \delta \text {-Untereinheit) } \\
\text { 17p13-p12 ( } \epsilon \text {-Unterein- } \\
\text { heit) (über } 50 \text { verschiedene } \\
\text { Mutationen bekannt) }\end{array}$ \\
\hline
\end{tabular}


Die Therapie gestaltet sich meist schwierig. Azetylcholinesterasehemmer sind nur bei einigen Syndromen wirksam (z.B. beim Azetylcholinrezeptormangel-Syndrom). Bei einem Mangel an Azetylcholinesterase können sie sich dagegen eher negativ auswirken.

Kalziumantagonisten können bei verlängerter Öffnungszeit der Kalziumkanäle des Azetylcholinrezeptors Symptome lindern.

\section{Lambert-Eaton-Syndrom Klinik}

Hauptsymptom ist eine Schwäche und abnorme Ermüdbarkeit der Rumpf- und bevorzugt der proximalen Extremitätenmuskulatur. Durch eine Beteiligung des autonomen Nervensystems werden z.B. auch Mundtrockenheit (bei 74\% der Patienten), Impotenz, Obstipation, Harnverhalt beklagt. Charakteristisch ist ein initialer Kraftzuwachs bei maximaler Willkürinnervation. Bei anhaltender Kraftanstrengung lässt die Kraft dann jedoch wieder nach.

\section{Pathogenese}

Das Lambert-Eaton myasthene Syndrom (LEMS) ist wie die Myasthenia gravis eine erworbene $\mathrm{Au}-$ toimmunerkrankung. Ursache der Symptomatik sind Antikörper gegen spannungsabhängige KalziumKanäle der präsynaptischen Membran an der neuromuskulären Synapse (voltage-gated calcium channel, VGCC) (Abb. 1). Die Antikörperbindung hat zur Folge, dass die spannungsabhängigen KalziumKanäle in ihrer Funktion gestört werden, folglich der Kalzium-Einstrom in die präsynaptische Nervenendigung reduziert und letztlich die Erregungsübertragung an der motorischen Endplatte vermindert wird.

In $60 \%$ der Patienten ist das LEMS eine paraneoplastische Erkrankung (meist Assoziation mit kleinzelligem Bronchial-Karzinom). Bei ca. 85\% der Patienten mit LEMS sind die VGCCAntikörper nachweisbar. Bei den übrigen 15\% kennt man die Ursache noch nicht; im Tierversuch konnte gezeigt werden, dass Serum von „seronegativen“ Patienten mit LEMS die neuromuskuläre Übertragung stört. Ein Antikörper wird als Aus- löser dieser Störung vermutet, konnte bisher jedoch noch nicht identifiziert werden (14).

\section{Diagnostik}

Der Nachweis der VGCC-Auto-Antikörper bestätigt die Verdachtsdiagnose (bei 85\% der Patienten nachweisbar). Gestützt wird die Diagnose durch elektrophysiologische Untersuchungen, insbesondere den LEMSTest (siehe Beitrag von M. Kornhuber in diesem Heft): Nach supramaximaler Nervenstimulation ist initial ein deutlich reduziertes Muskelsummenaktionspotenzial darstellbar, welches nach kurzer maximaler Willkürinnervation deutlich (>100\%) ansteigt (Inkrement).

Wenn die Tumorsuche ohne Tumornachweis verlief, sollten dennoch Kontrolluntersuchungen folgen (halbjährlich für drei Jahre, dann jährlich bis fünf Jahre), da das LEMS dem Tumornachweis lange vorausgehen kann.

\section{Therapie}

Mit der Therapie des Tumors bessern sich bei der paraneoplastischen Form des LEMS die Symptome; bei einem Tumorrezidiv ist auch mit einer erneuten Verschlechterung des LEMS zu rechnen.

3,4-Diaminopyridin stellt eine symptomatische Therapieoption dar (15 bis $50 \mathrm{mg} / \mathrm{d}$ ). Die Substanz verlängert die Dauer des präsynaptischen Aktionspotenzials durch Blockade des nach außen gerichteten Kaliumstroms, vergrößert den KalziumEinstrom in die Nervenendigung und dadurch die Menge des freigesetzten Azetylcholins. Es ist sowohl auf die motorischen als auch auf die autonomen Symptome wirksam.

Steroide werden bei sowohl paraneoplastischen als auch nicht paraneoplastischen LEMS eingesetzt, wenn die symptomatische Therapie nicht ausreicht.

Azathioprin kann eingesetzt werden bei nicht-paraneoplastischem LEMS, wenn die symptomatische Therapie nicht zu befriedigenden Ergebnissen führt.

Immunglobuline (IVIG) und Plasmapherese stehen als „ReserveTherapien“ zur Verfügung $(12,13)$. Summary
Diseases with neuromuscular transmission defect (NMTD) are myasthenia gravis with a postsynaptic defect and Lambert-Eaton myasthenic syndrome (LEMS) with a presynaptic defect. Autoantibodies are crucial in the pathogenesis of both disorders. Congenital heterogenous myasthenic syndromes are a group of hereditary diseases. In the last few years knowledge about these syndromes improved rapidly. Diseases with a neuromuscular transmission defect usually present with proximal weakness of the limbs, ptosis and external opthalmoplegia. Symptoms in myasthenia gravis are worsening during exercise and during the day. LEMS symptoms are not restricted to proximal limb weakness but also autonomic symptoms like dry mouth and constipation. Therapeutic options include acetylcholine esterase blocker in myasthenia gravis and some forms of congenital myasthenic syndromes, 3,4-diaminopyridine in LEMS and immunosuppressants in both.

\section{Key Words}

myasthenia gravis - congenital myasthenic syndrome - LambertEaton myasthenic syndrome (LEMS) - neuromuscular transmission defect (NMTD)

\section{Literatur}

1. Baggi F, Andreetta F, Antozzi C, Simoncini O, Confalonieri P, Labeit S, Cornelio F, Mantegazza R. Anti-titin and antiryanodine receptor antibodies in myasthenia gravis patients with thymoma. Ann N Y Acad Sci 1998; 841: 538-541

2. Bever CT, Aquino AV, Penn AS, et al. Prognosis of ocular myasthenia. Ann Neurol 1983; 14: 516-519

3. Congenital myasthenic syndromes: gene mutations. Neuromusc Disord 2003; 13: $115-119$

4. Gautel M, Lakey A, Barlow DP, Holmes Z, Scales S, Leonard K, Labeit S, Mygland A, Gilhus NE, Aarli JA. Titin antibodies in myasthenia gravis: identification of a major immunogenic region of titin. Neurology 1993; 43: $1581-1585$

5. Grob D, Brunner N, Namba T. The natural course of myasthenia gravis and effect of therapeutic measures. Ann N Y Acad Sci 1981; 377: 652-669

6. Hoch W, McConville J, Helms S, Newsom-Davies J, Melms A, Vincent A. Auto-antibodies to the receptor tyrosine kinase MuSK in patients with myasthenia gravis without acetylcholine receptor antibodies. 
Nat Med 2001; 7: 365-368

7. Kupersmith MJ, Moster M, Bhuiyan S, et al. Beneficial effects of corticosteroids on ocular myasthenia gravis. Arch Neurol 1996; 53: 802-804

8. Lastoria S, Vergara E, Palmieri G, et al. In vivo detection of malignant thymic masses by indium-111-DTPA-D-octreotide scintigraphy. J Nucl Med 1998; 39: 634-639

9. Lindner A, Schalke B, Toyka KV. Outcome in juvenile-onset myasthenia gravis: a retrospective study with long-term followup of 79 patients. J Neurol 1997; 244: 515-520

10. Melms A, Hohlfeld R. Zur Ätiologie und Pathogenese der Myasthenia gravis. Akt Neurol 1998; 25: S39-S41

11. Molnar J, Szobor A. Myasthenia gravis: effect of thymectomy in 425 patients: a 15years experience. Eur J Cardiothorac Surg 1990; $4: 8$

12. Motomura M, Hamasaki S, Nakane S, Fukuda T, Nakao YK. Apheresis treatment in Lambert-Eaton myasthenic syndrome. Ther Apher 2000; 4: 287-290

13. Muchnik S, Losavio AS, Vidal A, Cura L, Mazia C. Long-term follow-up of LambertEaton syndrome treated with intravenous immunglobulin. Muscle Nerve 1997; 20: 674-678

14. Nakao YK, Motomura M, Fukudome T, et al. Seronegative Lambert-Eaton myasthenic syndrome: study of 110 Japanese patients. Neurology 2002; 59: 1773-1775

15. Schumm M, Wiethölter H, Fateh-Moghadam A, Dichgans J. Thymectomy in myasthenia with pure ocular symptoms. J Neurol Neurosurg Psychiatry 1985; 48: 332-337 16. Schumpelick V, Janzen R. Thymektomie bei Myasthenia gravis. Dtsch Med Wochenschr 1984; 109: 1166-1172

17. Toyka KV, Becker T, Fateh-Moghadam A et al. Die Bedeutung der Bestimmung von Antikörpern gegen Azetylcholin-Rezeptoren in der Diagnostik der Myasthenia gravis. Klin Wochenschr 1979; 57: 937-942

18. Vincent A, Newsom-Davies J. Azetylcholine receptor antibody as a diagnostic test for myasthenia gravis: results in 153 validated cases and 2967 diagnostic assays. J Neurol Neurosurg Psychiatry 1985; 48: 1246-1252

19. Voltz RD, Albrich WC, Nägele $A$, Schumm F, Wick M, Freiburg A, Gautel M, Thaler T, Aarli J, Kirchner T, Hohlfeld R. Paraneoplastic myasthenia gravis: Detection of anti-MGT30 (titin) antibodies predicts thymic epithelial tumor. Neurology 1997; 49 : 1454-1457

20. Wekerle H, Hohlfeld R, Ketelsen UP, Kalden JR, Kalies I. Thymic myogenesis, T-lymphocytes and the pathogenesis of myasthenia gravis. Ann N Y Acad Sci 1981; 377 : 455-476

\section{Korrespondenzanschrift:}

Dr. med. Katharina Eger

Klinik und Poliklinik für Neurologie der Martin-Luther-Universität Halle-Wittenberg

E.-Grube-Str. 40

06097 Halle/Saale

katharina.eger@medizin.uni-halle.de

\section{Nervensachen. Perspektiven zu Geist, Gehirn und Gesellschaft}

Manfred Spitzer: Nervensachen. Perspektiven zu Geist, Gehirn und Gesellschaft. Schattauer-Verlag, 2003. 368 Seiten, gebunden, 29,95€. ISBN 37945-2202-8

D ieses Buch ist ein Ereignis, eine geradezu fabelhafte Sammlung von neuen Erkenntnissen unseres Faches Nervenheilkunde in Gestalt von „Geschichten“, die sehr einprägsam und hervorragend geschrieben sind. Es ist vielleicht etwas zu flott, Ausflüge in die Politik sind nicht unbedingt wichtig, insbesondere was die Universität anlangt, und wahr- scheinlich wird gelegentlich über das Ziel hinaus geschossen, z.B. wenn sich teleologische Gedanken in den Vordergrund drängen, (Seite 335, wofür kann eine depressive Phase gut sein?).

Aber insgesamt Respekt, wer so viel weiß und das Fach so gut übersieht und auch noch eindrucksvoll schreiben kann, ist des Lobes wert. Dem Buch ist große Verbreitung zu wünschen, insbesondere geht es die an, die auch Bücher schreiben wollen, um zu lernen, wie man das zu machen hat.

Prof. F. Reimer, Weinsberg

\section{Die Aufmerksamkeitsdefizit-Hyper- aktivitätsstörung bei Erwachsenen}

Johanna Krause, Klaus-Henning Krause: ADHS im Erwachsenenalter. Die Aufmerksamkeitsdefizit-Hyperaktivitätsstörung bei Erwachsenen. Schattauer-Verlag, Stuttgart, 2003. 160 Seiten, ca. 12 Abb., kart., 29,95€. ISBN: 3-7945-2243-5.

D ie Aufmerksamkeitsdefizit-Hyperaktivitätsstörung (ADHS) ist bei Kindern und Jugendlichen relativ gut bekannt, bei Erwachsenen bisher noch nicht ausreichend beschrieben, obwohl man davon ausgehen konnte, dass es diese Störung auch im Erwachsenenalter gibt. Die beiden Autoren haben sich die Mühe gemacht, alles zu diesem Thema Bekanntes zusammen zu tragen und in einer Monographie niederzulegen. Den Autoren ist dafür zu danken, umso mehr, als sie diese Arbeit quasi nebenbei geleistet haben ohne den im Allgemeinen nötigen Hintergrund einer großen Klinik.

Das Krankheitsbild wird nachvollziehbar beschrieben, die Aussagen der Autoren sind klar und deutlich. Bemängeln müsste man lediglich, dass wie immer in solchen Fällen einer Neuentdeckung einer
Krankheit oder Störung ein wenig überzogen wird. Das aber wird die Zukunft klären und es wird sich vermutlich herausstellen, dass es besser ist, mit dieser neuen Störung umzugehen, als die Einzeleffekte mit verschiedenen Persönlichkeitsstörungen zusammen zu bringen. Problematisch ist der Vorschlag, bei Erwachsenen Stimulanzien zu verordnen, weil meiner Ansicht nach dann die erhöhte Gefahr des Missbrauchs besteht und die Folgen der Behandlung u.U. gravierender sind als die Grundkrankheit selber.

Bei den tiefenpsychologisch fundierten Verfahren ist es sehr die Frage, ob daraus tatsächlich ein Nutzen erwachsen kann. Bei Kindern zumindest sind solche Verfahren nicht unbedingt zu empfehlen. Bei Erwachsenen aus den selben Gründen ebenfalls nicht.

Das Buch ist ein Standardwerk, es wird in keiner Fachbibliothek fehlen können. Vermutlich werden sich in der nächsten Ausgabe weitere Gesichtspunkte ergeben. Auf jeden Fall ist den Autoren für ihre Arbeit sehr zu danken.

Prof. F. Reimer, Weinsberg 